\title{
Kinetics of Photo-Induced Chemiluminescence Decay from Polymers
}

\author{
By Keith R. MILLINGTON* and George MAURDEV
}

Previous studies showed that the decay of photo-induced chemiluminescence (PICL) in polymers does not obey classical first or second order kinetics. This study demonstrates that plotting PICL second-order decay from three fibrous polymers (polypropylene, polyamide 6 and wool keratin) in a modified form gives excellent linear plots. The existence of a significantly less reactive fraction of macroperoxy radicals in heterogeneous polymers can explain the deviation from classical behaviour and this fraction can be quantified from the gradient of the modified plots. This unreactive fraction could be due to either dispersive or diffusive effects in a disordered heterogeneous polymer medium. Both dispersive and diffusive models give very good fits to the PICL decay profiles using three fitting parameters.

KEY WORDS: Polymer Luminescence / Photo-Induced Chemiluminescence / Macroperoxy Radical / Luminescence Decay Kinetics /

Organic materials, including synthetic and biopolymers, emit weak chemiluminescence (CL) when they undergo oxidative degradation. The phenomenon was first observed in 1961 from polymers heated in air by Ashby, ${ }^{1}$ who understood the possibility of using CL as a method for measuring polymer stability. CL emission originates from side reactions of the peroxy radical and hydroperoxide intermediates formed during the autoxidation chain reaction originally developed by Bolland and $\mathrm{Gee}^{2}$ and now generally applied to numerous polymers and biomaterials. Since 1961 a large number of CL studies have been reported on the thermal oxidation of commercially significant polymers, in particular polypropylene, and CL has become an important means of following the initial stages of polymer oxidation. Isothermal CL experiments on commercial polymers in oxygen or air at elevated temperatures allow measurement of the oxidation induction time (OIT), which can be used to determine the working lifetime of additives such as antioxidants and to optimise additive formulations. ${ }^{3}$ A number of recent reviews of polymer CL and its applications have been published. ${ }^{4-6}$

Most polymers also emit weak luminescence after a brief exposure to ultraviolet (UV) or visible light. When polymers are irradiated in an inert atmosphere (nitrogen or argon) and all excited electronic states are allowed to decay via fluorescence, phosphorescence or radiationless processes before changing the surrounding atmosphere to oxygen, a burst of photo-induced chemiluminescence (PICL) emission is emitted which decays slowly for periods of up to an hour.

PICL originates from the binary reaction of macroperoxy radicals formed in the polymer when the irradiated material is exposed to oxygen. The PICL intensity is related to the population of polymer free radicals and the PICL decay profile with time provides information on their lifetime, mobility and reactivity. These properties are clearly important ones in studies on the photodegradation of polymers and the PICL technique can provide insight into the photooxidation mechanisms, optimisation of additive systems and allow the prediction of a material's lifetime well before any physical damage can be observed or measured. The latter is particularly important in the conservation of valuable artefacts made from polymer and biopolymer materials exposed to light during exhibition in museums and galleries. A preliminary application of PICL in the field of conservation has been described which demonstrates the sensitivity of the technique. In a study on silk fabric, exposure to a single photographic flash can generate significant PICL emission. ${ }^{7}$

Whilst there have been a great many chemiluminescence studies on the thermal oxidation of polymers, in particular on polyolefins, only a handful of mechanistic studies on polymer PICL have been reported. Work on the synthetic polymers polystyrene, ${ }^{8}$ polypropylene (PP),${ }^{9,10}$ polyethylene $(\mathrm{PE})^{11}$ and polyamide (PA) 6,6 $6^{12}$ has been reported, and we have recently carried out studies on PICL emission from the fibrous proteins wool and feather keratin, silk fibroin and collagen. ${ }^{13}$ In our study, attempts to fit first or second order kinetics to the PICL decay of fibrous proteins resulted in highly non-linear plots, ${ }^{13}$ this behaviour also being observed for $\mathrm{PP}^{9}$ and PA6, $6,{ }^{12}$ although PE produces a linear plot of $\mathrm{I}^{-1 / 2} \mathrm{CL}$ against time, characteristic of 2 nd order kinetics. ${ }^{11}$

In this paper we have further examined the emission of PICL from three different polymers, commercial samples of PP, PA6 and wool keratin, over a range of temperatures from $40-140{ }^{\circ} \mathrm{C}$ following exposure to UVA $(320-400 \mathrm{~nm})$ radiation. In particular we have sought to develop a common mechanism capable of describing the PICL decay kinetics in solid polymers.

\section{EXPERIMENTAL}

\section{Materials}

Polypropylene fabric used for commercial filtration of 
alkali-solubilised alumina was supplied by Clear Edge Filtration, Moorabbin, Australia. Polyamide 6 fabric (ripstop nylon) was supplied by Melba Industries, Thomastown, Australia. Lightweight $100 \%$ Merino wool challis fabric was obtained from Armitage Ltd, Bradford, UK. The two synthetic fibres contained additives, with PA6 also containing titanium dioxide as a delustrant. Wool fabric was scoured using an aqueous nonionic surfactant solution $(1 \% \mathrm{w} / \mathrm{v})$ at $50^{\circ} \mathrm{C}$ and thoroughly rinsed with purified water several times before drying.

\section{Photo-Induced Chemiluminescence (PICL)}

A Lumipol 3 thermal CL instrument was reversibly modified to allow in situ irradiation with selected wavelengths from a medium-pressure mercury arc (Lumatec SUV-DC, Lumatech $\mathrm{GmbH}$, Germany) for PICL studies. Details of the PICL instrument and the modification have been described previously. ${ }^{13}$ A circular sample $8 \mathrm{~mm}$ in diameter was cut from each fabric using a sharpened punch and placed into an aluminium sample pan in the instrument sample cavity. The chemiluminescence baseline was stabilised by equilibrating each sample in a nitrogen atmosphere before exposure using a gas flow rate of $\sim 200 \mathrm{~cm}^{3} /$ minute at constant temperature. Samples were irradiated with UVA light in $\mathrm{N}_{2}$ for $30 \mathrm{~s}$ (PA6 and wool) or $2 \mathrm{~min}$ (PP). At the end of the irradiation period an intense, rapidly decaying emission was observed in $\mathrm{N}_{2}$ which includes phosphorescence emission and charge recombination chemiluminescence from the irradiated sample. This feature was always present even in the absence of a sample, and originates in part from irradiation of the metal components in the sample cavity. This behaviour was previously observed by George et al., ${ }^{12}$ who devised a protocol for separating PICL from other luminescence that may be present. At a fixed time after cessation of irradiation (one minute for PA6 and wool, 2 min for PP), the atmosphere was switched from nitrogen to oxygen, resulting in a burst of PICL that decayed far more slowly than the peak observed in nitrogen.

The UVA $(320-400 \mathrm{~nm})$ radiation range was chosen for two reasons. Firstly, most of the terrestrial UV radiation from sunlight lies in the UVA region $(\sim 5.1 \%$ UVA, $\sim 0.3 \%$ UVB) and UVA is known to cause photooxidation in polymers. ${ }^{14}$ Secondly light from the Lumatech source used in our PICL apparatus is transferred to the sample cavity via a liquid light guide, and the liquid used is sensitive to UVB $(280-320 \mathrm{~nm})$ and UVC (200-280 $\mathrm{nm}$ ) radiation, resulting in cavitation within the light guide and loss of efficiency.

\section{Computer Modelling}

Curve fitting and computer modelling studies were carried out using SigmaPlot Version 10 (Systat Software Inc, Chicago, USA).

\section{RESULTS AND DISCUSSION}

\section{PICL Decay Profiles and Preliminary Kinetic Analysis}

The corrected PICL decay curves for PA6, PP and wool keratin in oxygen over a range of temperatures are shown in
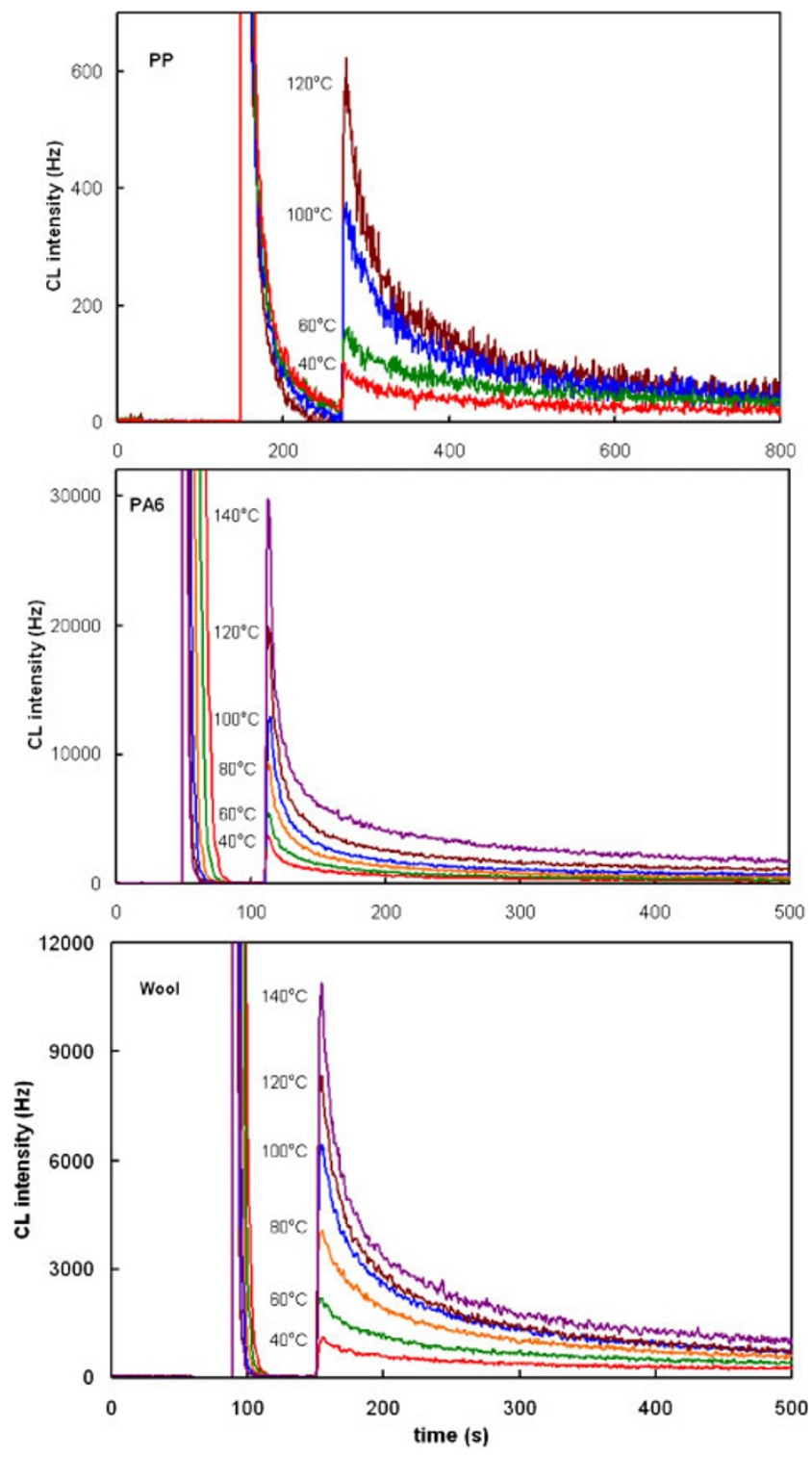

Figure 1. Corrected PICL decay profiles of PP, PA6 and wool keratin fabrics following exposure to UVA radiation at various temperatures. The profiles were corrected to remove the contribution due to thermal oxidation at each temperature.

Figure 1. Correction was made to these curves to remove the contribution due to thermal oxidation of the polymers by subtracting the trace obtained using an identical experimental protocol at the appropriate temperature in the absence of light, as previously described. ${ }^{13}$ PICL emission from PP is significantly weaker than PA6 or wool, since pure PP contains no chromophores capable of absorbing UVA radiation, so absorption is probably due to the presence of additives or impurities in the polymer. Both PA6 and wool absorb UVA far more strongly. In addition to any additives and the $\mathrm{TiO}_{2}$ delustrant which absorb in this region, PA6 is known to contain traces of UV-absorbing impurities, such as carbonyl and pyrrolyl groups, and also conjugated oligoenimines formed via Schiff bases following exposure to heat during thermal processing. ${ }^{15,16}$ Wool keratin absorbs UVA due to the presence of tryptophan 


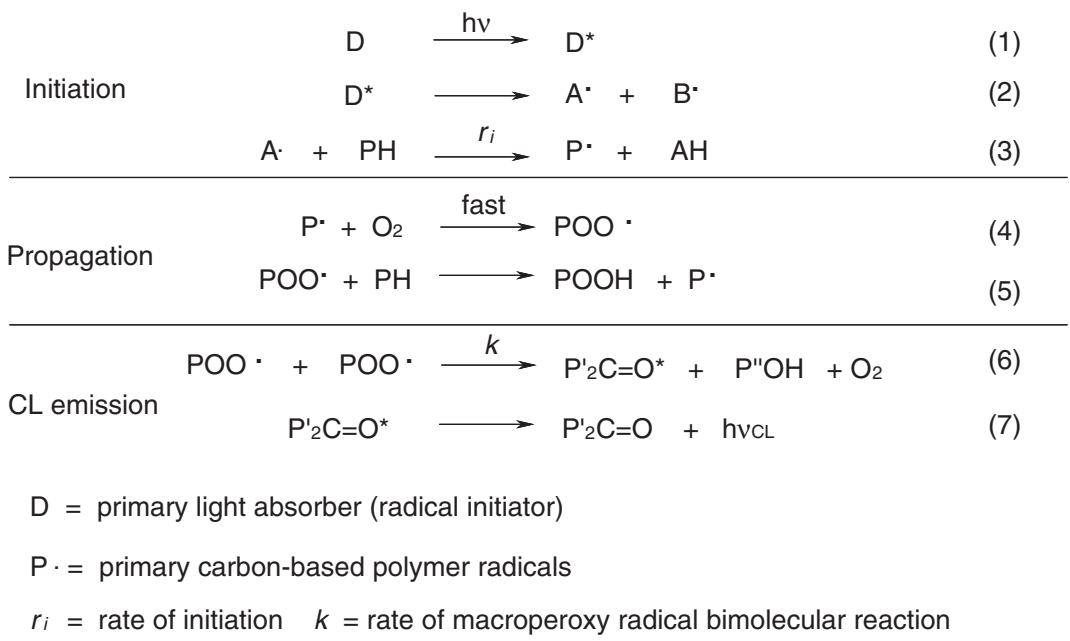

Scheme 1. Generally accepted mechanism for $\mathrm{CL}$ emission from irradiated polymers (Russell mechanism).

(Trp) residues and the natural cream chromophores of wool, the nature of which is currently unknown but may include Trp oxidation products such as $\beta$-carbolines and kynurenines. ${ }^{17}$

Emission of PICL from polymers is dependent on the presence of oxygen, and for hydrocarbon polymers the accepted dominant mechanism involves a bimolecular reaction between two adjacent polymer macroperoxy radicals via an excited carbonyl intermediate, as shown by eq 6 in Scheme 1.

In an ideal homogeneous system where the free radicals are not constrained, the decay of CL emission following irradiation would be expected to demonstrate simple second-order kinetics according to the equation

$$
\frac{d\left[R O_{2}\right]}{d t}=-k\left[R O_{2}\right]^{2}
$$

the solution to which is, converting to CL intensities

$$
\left(\frac{I o}{I}\right)^{1 / 2}=1+\left(k r_{i}\right)^{1 / 2} t
$$

where $\mathrm{I}_{\mathrm{o}}$ and $\mathrm{I}$ are the CL intensity at $\mathrm{t}=0$ (admission of $\mathrm{O}_{2}$ ) and $\mathrm{t}, \mathrm{k}$ is the rate constant for the bimolecular reaction, and $r_{i}$ is the rate of initiation of oxidation as shown in Scheme 1. ${ }^{12}$ Eq 9 can also be written as:-

$$
\frac{1}{\sqrt{I}}=\left(\frac{k}{\phi}\right)^{1 / 2} t+\frac{1}{\sqrt{I o}}
$$

where $\varphi$ is the overall efficiency of the formation and emission of the excited ketone produced via eq 6 . Therefore a plot of $1 / \sqrt{\mathrm{I}}$ against time should give a linear plot. Figure 2 shows the second order plots for PP, PA6 and wool keratin. In all cases there is a clear deviation from linearity, particularly during the initial period of CL decay. The high scatter apparent in the plots for PP is due to the much lower intensity of PICL from PP irradiated with UVA compared with PA6 and wool, due to the much lower absorption of PP in the $320-400 \mathrm{~nm}$ range. Our previous work showed that fitting 1 st order kinetics to the PICL decay of wool gave an inferior fit to that shown in Figure $2^{13}$ and this was confirmed for PP and PA6 (data not shown).
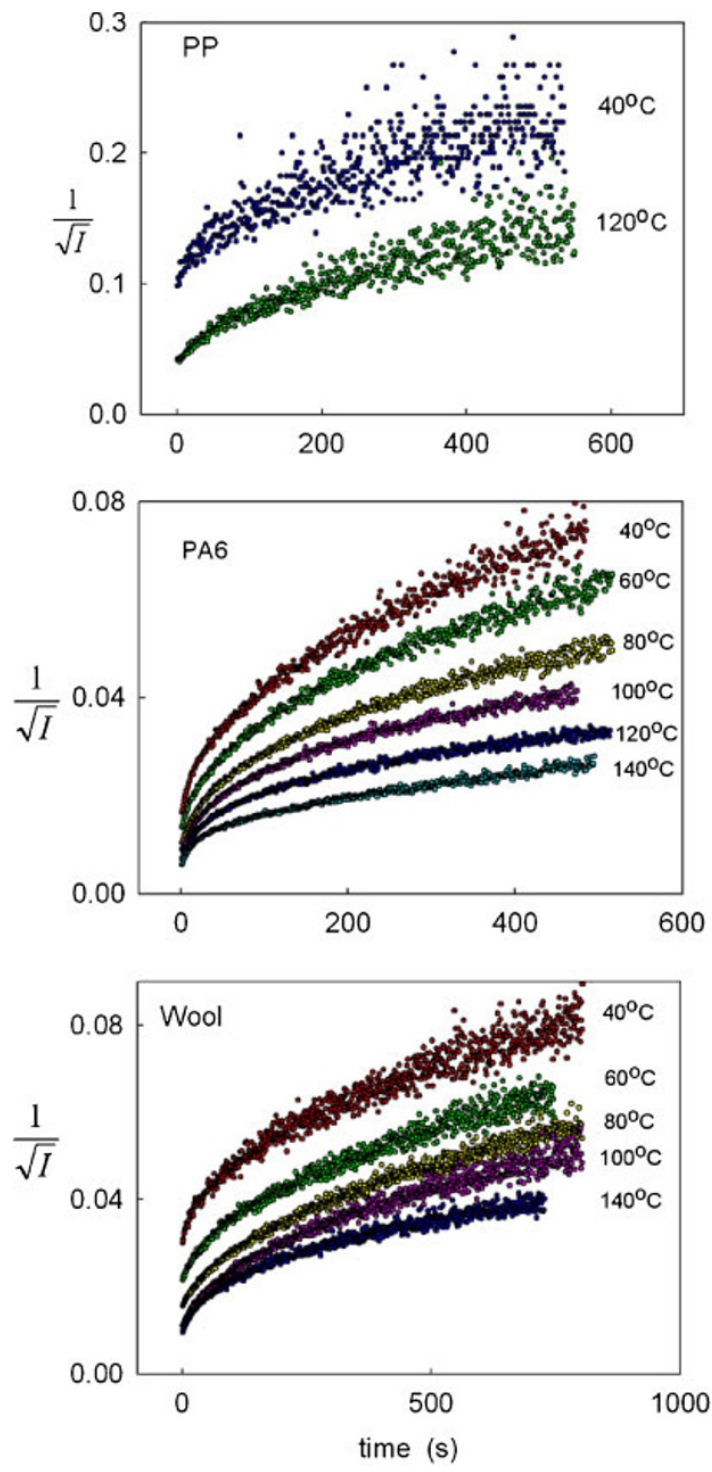

Figure 2. Second order plots of PICL decay from PP, PA6 and wool keratin at various temperatures. 
This non-linear 2nd order decay behaviour has been observed previously in PICL studies on $\mathrm{PP}^{9}$ and PA6,6. ${ }^{12}$ Similar deviations from 2nd order kinetics have been widely observed in ESR studies on the decay of free radicals formed by $\mathrm{UV}$, electron beam, $\gamma$ - or X-radiation trapped in polymer matrices. ${ }^{18}$ From an ESR study of the macroperoxy radical propagation stages (eqs 4 and 5) in the presence of isotopically labelled oxygen at low temperature in PP films, Carlsson et al. ${ }^{19}$ showed that a fraction of the $\mathrm{PO}_{2}$ radicals behaved as if they were trapped in motionally-resticted domains, and therefore unable to propagate. They concluded that macroperoxy radical reactivity is controlled by mobility factors which make $\mathrm{PO}_{2}$. radicals either highly reactive to propagation and termination, or extremely unreactive. They also showed that all of the macroalkyl radicals $(\mathrm{P})$ formed after $\gamma$-irradiation reacted very rapidly with $\mathrm{O}_{2}$, and so must be readily accessible to $\mathrm{O}_{2}$ either because the crystalline domains in PP contain a large number of defects or due to rapid migration of $\mathrm{P}$ from crystalline to amorphous regions by a hydrogen-transfer process. ${ }^{19}$

\section{Second-Order Kinetic Analysis Assuming an Unreactive Free Radical Fraction}

The three polymers shown in Figures 1 and 2 have very similar decay profiles, which may suggest that PA6 and wool keratin behave similarly to PP in terms of having a fraction of macroperoxyradicals with highly restricted radical mobility. Some years ago similar deviations from second-order free radical decay behaviour were observed in ESR experiments on $\gamma$-irradiated polymers performed at low temperatures. The group led by Malcolm Dole studied the decay of free radicals in a number of polymers, including poly (1,4 butadiene $),{ }^{20} \mathrm{PP}^{21}$ and epoxy resins. ${ }^{22}$ The ESR signal intensity of these polymers irradiated at $77 \mathrm{~K}$ and then annealed to a higher temperature to allow reactions to occur decreased in a very similar manner to the PICL intensities of PP, PA6 and wool shown in Figure 1 and gave very poor fits to the standard 2nd order equation, similar to those presented here in Figure 2.

Dole rearranged the form of the standard second-order rate equation

$$
\frac{1}{c}-\frac{1}{c_{o}}=k_{2} t
$$

to give

$$
\frac{t}{c_{o}-c}=\frac{t}{c_{o}}+\frac{1}{c_{o}^{2} k_{2}}
$$

where $\mathrm{c}_{\mathrm{o}}$ and $\mathrm{c}$ are the esr signal intensities at $t=0$ and $t$, and $k_{2}$ is the 2nd order rate constant. A plot of $t /\left(c_{\mathrm{o}}-c\right)$ against $t$ was used to replot the ESR intensity decay data, and this resulted in an excellent linear relationship for a number of different $\gamma$-irradiated polymers. ${ }^{23}$

We applied a similar approach to rearranging eq 10, which using CL intensities gives:-

$$
\frac{t}{\sqrt{I o}-\sqrt{I}}=\frac{t}{\sqrt{I o}}+\frac{1}{I o}\left(\frac{\phi}{k}\right)^{1 / 2}
$$
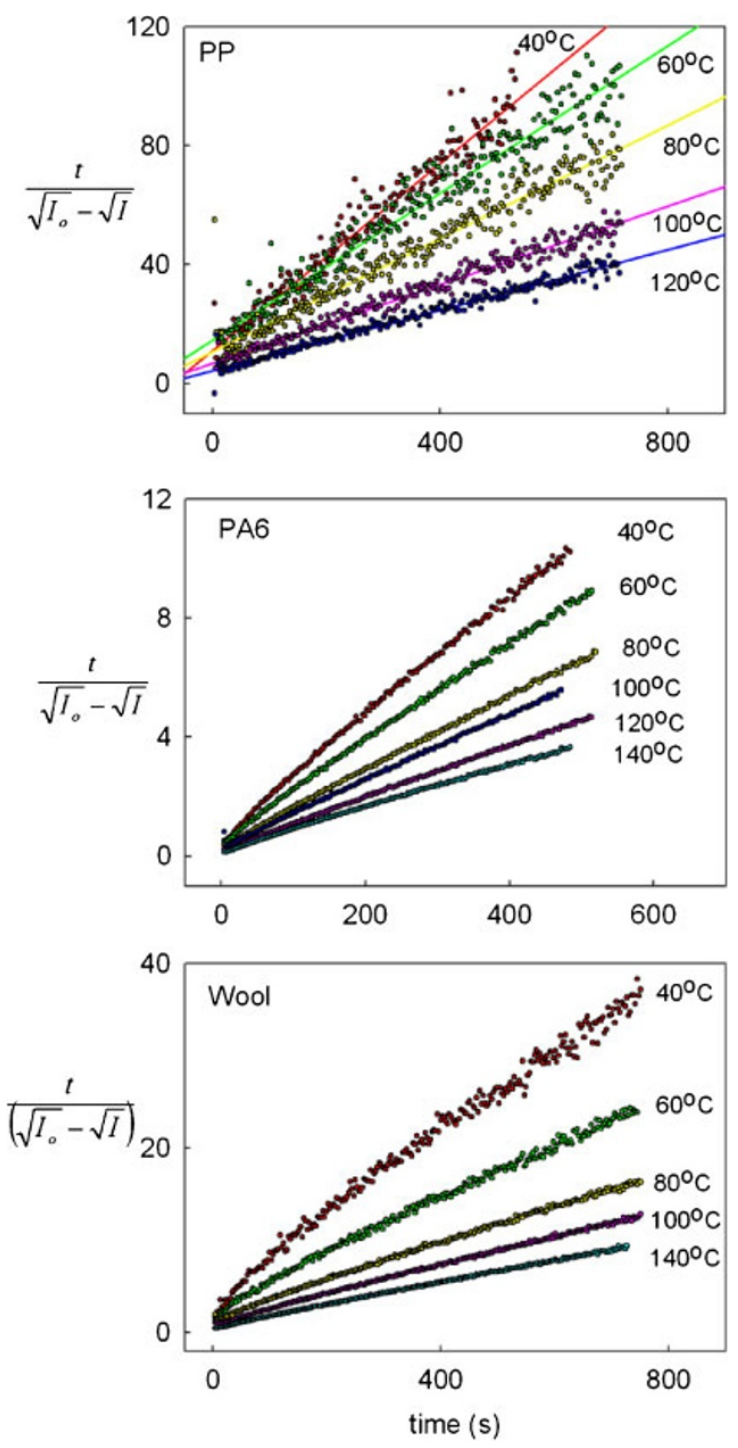

Figure 3. Modified 2nd order 'Dole plots' for PP, PA6 and wool keratin at various temperatures from eq $14 .{ }^{16,17}$

Using data from the PICL decay profiles of PP, PA6 and wool keratin derived from Figure 1, and substituting into eq 13 resulted in the excellent linear plots shown in Figure 3.

Comparing the plots in Figure 3 to the conventional 2nd order plots of CL decay in Figure 2 shows that the model used to obtain the former is greatly superior for the three disparate polymer systems examined in our study. Dole discussed the reason why plotting eq 12 gave linear plots, whereas the normal second order relationship (eq 11) gave highly non-linear ones. Any free radicals trapped in an irradiated polymer can only disappear by reaction with other free radicals in a second-order process, but Dole proposed that a fraction of the radicals may be formed in regions of the polymer where they cannot react. ${ }^{20}$ This unreactive radical fraction is a similar concept to the existence of macroperoxy radicals trapped in motionallyresticted domains proposed two years later by Carlsson et al. ${ }^{19}$

By making the concentration of unreactive radicals equal to A, Dole obtained 


$$
\frac{1}{c-A}-\frac{1}{c_{o}-A}=k_{2} t
$$

On rearrangement this becomes

$$
\frac{t}{c_{o}-c}=\frac{t}{c_{o}-A}+\frac{1}{\left(c_{o}-A\right)^{2} k_{2}}
$$

From eq $15, t /\left(c_{\mathrm{o}}-c\right)$ against t plots will clearly remain linear regardless of the concentration of unreactive radicals present. By further rearrangement the fraction of unreactive radicals $\left(A / c_{0}\right)$ is given by

$$
\frac{A}{c_{o}}=1-\frac{1}{m c_{o}}
$$

where $\mathrm{m}=$ the gradient of the plot.

Since

$$
I_{o}=\phi k\left[R O_{2}\right]^{2}=\phi k c_{o}^{2}
$$

for polymer PICL studies, this becomes

$$
\frac{A}{\sqrt{I_{o}}}=\frac{1}{\sqrt{\phi k}}-\frac{1}{m \sqrt{I_{o}}}
$$

Since $I_{\mathrm{O}}$ is known from the data, the fraction of unreactive radicals $\mathrm{A}$ in each material can be determined from the gradient of the plot. At $40^{\circ} \mathrm{C}$, the fraction of unreactive radicals in PP is 0.38, wool 0.32 and PA6 0.18. As the temperature increases the fraction of unreactive radicals decreases as expected for PP and wool but not for PA6, as shown in Figure 4. The commercial PA6 fabric used here contains unknown additives and $\mathrm{TiO}_{2}$ delustrant which may explain its unexpected behaviour. It is interesting that the linearity of the Dole plots appears to be unaffected by the presence of additives.

The crystallinity fractions of isotactic PP, PA6 and wool fibres quoted in the literature are PP $0.45-0.5,{ }^{24}$ PA6 $0.4^{25}$ and wool $0.25^{26}$ and crystallinity does not vary significantly with temperature except close to the melting point, so it appears that the fraction of unreactive macroradicals formed by exposure to UVA is not related to polymer crystallinity.

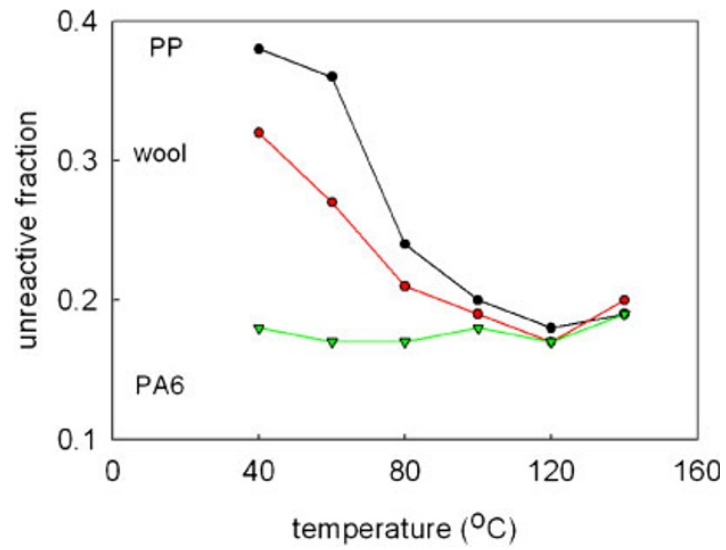

Figure 4. Plot of fraction of unreactive macroperoxy radicals in PP, PA6 and wool against temperature.
However the size distribution of polymer crystallites in these polymers is uncertain, and significant differences would certainly affect the rate at which radicals may migrate from crystalline into amorphous regions. Defect-free polymer crystallites are thought to be impermeable to oxygen, so it is interesting that ESR studies show that the free radical populations in irradiated polymers with high crystalline fractions, such as isotactic PP, decrease to zero on standing. ${ }^{9}$

\section{Dispersive Model for PICL Decay}

The high linearity of the 'Dole plots' in Figure 3 suggest that a dispersive kinetic model is applicable to the PICL decay for a diverse range of polymer materials such as those examined in this study. A more general equation for 2 nd order dispersive kinetics was described by Plonka ${ }^{18}$ and can be written as:-

$$
c^{-1}-c_{o}^{-1}=B \alpha^{-1} t^{\alpha}
$$

where $\alpha$ and $\mathrm{B}$ are constants.

Converting to PICL intensities using eq 17 gives:-

$$
\sqrt{\frac{\phi k}{I}}-\sqrt{\frac{\phi k}{I_{o}}}=\frac{B t^{\alpha}}{\alpha}
$$

or

$$
\frac{1}{\sqrt{I}}-\frac{1}{\sqrt{I_{o}}}=\frac{B t^{\alpha}}{\alpha \sqrt{\phi k}}=\frac{B^{\prime} t^{\alpha}}{\alpha}
$$

Including a non-reactive fraction $\mathrm{A}$, this becomes:-

$$
\left(\sqrt{I_{o}}-\sqrt{I}\right)^{-1}=\left(\sqrt{I_{o}}-A\right)^{-1}+\frac{\alpha}{\left(B^{\prime} t^{\alpha}\left(\sqrt{I_{o}}-A\right)^{2}\right)}
$$

Fitting this equation to the square root of the PICL decay for the three polymers examined in this study gave very good fits, as shown by the $r^{2}$ values for PA6 and wool shown in Figure 5, the noisier lower-intensity PP decay data having lower $r^{2}$ values.

Because eq 22 contains three fitting parameters, (A, B' and $\alpha$ ), the values of the unreactive radical fraction $\mathrm{A}$ derived from these plots for wool and PP do not show the stepwise decrease in A with temperature derived from the 'Dole plots' shown in Figure 4. Table I shows the values of kinetic parameters derived from the least-squares fitting, and the values of A for the dispersive model lie in the range $0.08-0.25$ showing no particular trends with temperature. The Dole plots are therefore clearly superior in having only one parameter, A.

Our intention in further studies is to look at PICL decay from some well characterised, additive-free polymers using the Dole approach. This will determine whether useful information on the effects of temperature on the non-reactive fraction A can be obtained and hence the effects of temperature on free radical mobility in polymers.

\section{Diffusion-Controlled Model for PICL Decay}

A recent ESR study on the free radical decay kinetics in a non-woven mat of carbohydrate-coated collagen fibres exposed to synchrotron radiation found that either a dispersive model or 

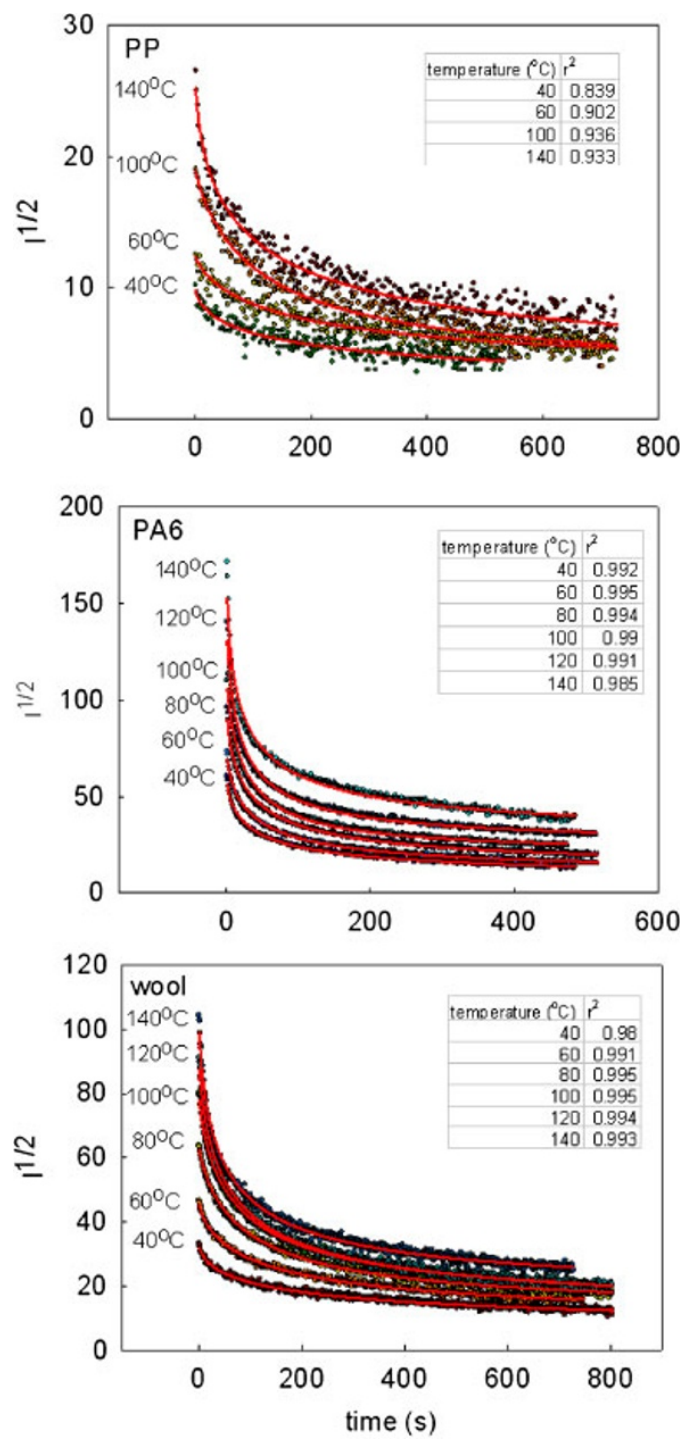

Figure 5. Results of fitting dispersive 2nd order kinetics (eq 22) to PICL decay curves from PP, PA6 and wool keratin.

a diffusion-controlled model could be fitted effectively to the decay profiles. ${ }^{27}$ The diffusion model described the radical reaction kinetics in terms of fast free radical decay on the fibre surface and a diffusion-limited slow decay of radicals formed inside the fibre. The dispersive model considered the molecular mobility and dispersivity of free radicals in a heterogeneous material, but only as a first-order decay process. We consider 2nd order radical-radical reactions to be more compelling, particularly for PICL emission.

We therefore also examined fitting a diffusive model to the PICL decay profiles. Hori et al. used a similar 2nd order diffusion-limited kinetic model to study the decay of isotactic peroxy radicals in $\operatorname{air}^{28}$ using the equation:-

$$
f(t)=\frac{1-A}{\left(1+b t^{1 / 2}+c t\right)}
$$

where $\mathrm{A}$ is the fraction of unreactive radicals and $\mathrm{b}$ and $\mathrm{c}$ are constants. It is important that diffusion here refers to diffusion
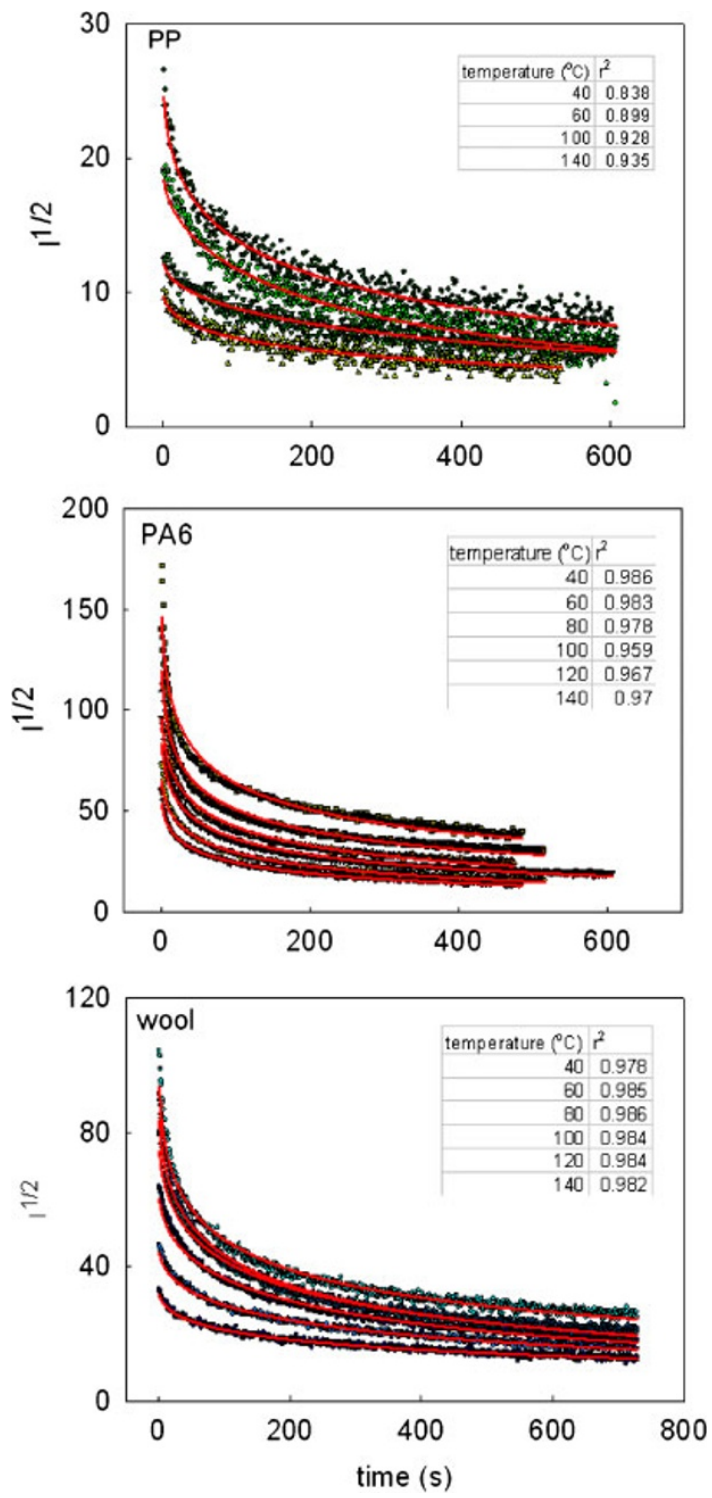

Figure 6. Results of fitting diffusion-limited kinetics (eq 23) to PICL decay curves from PP, PA6 and wool keratin.

of the macroperoxy radicals within the polymer, rather than oxygen. ${ }^{28}$ Fitting this equation to the PICL decay profiles produced the plots shown in Figure 6 with the fitted kinetic parameters shown in Table I. The $r^{2}$ values using the diffusive model are very similar to those found for the dispersive model in Figure 5, so both models fit the data well, as was the case for the ESR study on irradiated collagen of Gouk et al. ${ }^{27}$

Further studies are required to determine whether one of these models accurately describes the macroperoxy radical reaction kinetics in UV-irradiated materials. Currently we are examining the PICL from polymer films having a range of $\mathrm{O}_{2}$ permeabilities doped with the same dye at fixed concentration, to determine the effects of $\mathrm{O}_{2}$ access on PICL intensity. Initial results suggest that polymers having low $\mathrm{O}_{2}$ permeability (PVA and gelatin) have low PICL emission whereas those with high permeability (methyl and ethyl cellulose) produce intense PICL signals. 
Table I. Kinetic parameters obtained by fitting dispersive and diffusive models to PICL decay profiles for PP, PA6 and wool keratin

\begin{tabular}{|c|c|c|c|c|c|c|c|c|c|}
\hline \multirow{2}{*}{$\begin{array}{l}\text { Polymer temp. } \\
\qquad\left({ }^{\circ} \mathrm{C}\right)\end{array}$} & \multirow{2}{*}{$\sqrt{10}$} & \multicolumn{4}{|c|}{ Dispersive model $^{a}$} & \multicolumn{4}{|c|}{ Diffusive model ${ }^{b}$} \\
\hline & & $A^{\prime}$ & $\mathrm{B}^{\prime}$ & $\alpha$ & A (unreactive fraction) & $b$ & c & $A^{\prime}$ & A (unreactive fraction) \\
\hline \multicolumn{10}{|l|}{ PP } \\
\hline 40 & 10.2 & 1.30 & 0.0031 & 0.58 & 0.13 & 0.060 & $<0.0001$ & 0.27 & 0.03 \\
\hline 60 & 12.5 & 3.17 & 0.0015 & 0.78 & 0.25 & 0.057 & $<0.0001$ & 1.26 & 0.10 \\
\hline 100 & 19.1 & 2.98 & 0.0008 & 0.88 & 0.16 & 0.081 & 0.0013 & 3.33 & 0.17 \\
\hline 140 & 26.6 & 2.25 & 0.0016 & 0.63 & 0.08 & 0.115 & $<0.0001$ & 2.90 & 0.11 \\
\hline \multicolumn{10}{|l|}{ PA6 } \\
\hline 40 & 60.6 & 5.81 & 0.0011 & 0.68 & 0.10 & 0.198 & $<0.0001$ & 11.44 & 0.19 \\
\hline 60 & 73.4 & 8.57 & 0.0008 & 0.75 & 0.12 & 0.220 & $<0.0001$ & 19.04 & 0.26 \\
\hline 80 & 96.1 & 12.30 & 0.0007 & 0.77 & 0.13 & 0.232 & $<0.0001$ & 23.15 & 0.24 \\
\hline 100 & 109.9 & 19.77 & 0.0005 & 0.92 & 0.18 & 0.245 & $<0.0001$ & 34.04 & 0.31 \\
\hline 120 & 140.4 & 23.67 & 0.0006 & 0.80 & 0.17 & 0.207 & $<0.0001$ & 16.40 & 0.12 \\
\hline 140 & 171.6 & 24.61 & 0.0007 & 0.66 & 0.14 & 0.200 & $<0.0001$ & 20.00 & 0.12 \\
\hline \multicolumn{10}{|l|}{ wool } \\
\hline 40 & 33.3 & 3.44 & 0.0009 & 0.59 & 0.10 & 0.065 & $<0.0001$ & 1.66 & 0.05 \\
\hline 60 & 46.5 & 6.91 & 0.0006 & 0.71 & 0.15 & 0.083 & 0.0001 & 5.80 & 0.12 \\
\hline 80 & 63.7 & 10.70 & 0.0004 & 0.84 & 0.17 & 0.103 & $<0.0001$ & 11.41 & 0.18 \\
\hline 100 & 80.1 & 11.30 & 0.0004 & 0.78 & 0.14 & 0.139 & 0.0002 & 18.38 & 0.23 \\
\hline 120 & 91.2 & 10.08 & 0.0004 & 0.75 & 0.11 & 0.165 & 0.0002 & 22.35 & 0.25 \\
\hline 140 & 104.2 & 15.77 & 0.0005 & 0.73 & 0.15 & 0.142 & $<0.0001$ & 13.25 & 0.13 \\
\hline
\end{tabular}

a Dispersive model fitted using $f(t)=\sqrt{I_{0}}-\left(\frac{1}{\left(\sqrt{I_{0}}-A^{\prime}\right)}+\frac{\alpha}{B t^{\alpha}\left(\sqrt{I_{0}}-A^{\prime}\right)^{2}}\right)^{-1}$. ${ }^{b}$ Diffusive model fitted using $f(t)=\frac{\left(\sqrt{I_{0}}+A^{\prime}\right)}{\left(1+b t^{1 / 2}+c t\right)}$.

\section{CONCLUSIONS}

This study has shown that a relationship based on the expected 2nd order kinetics of a bimolecular reaction between macroperoxy radicals, but with a less reactive radical fraction present as described previously by Dole, ${ }^{22,23}$ can produce excellent linear plots for the decay of PICL from three disparate polymer systems over a range of temperatures. The fraction of less reactive radicals can be calculated from the plots and this fraction reduces with temperature as expected for PP and wool keratin, but not for PA6. The decay profiles can be fitted well using second-order dispersive and diffusive models with three fitting parameters. Further PICL studies are currently in progress to determine whether such behaviour is universal in additive-free UV-irradiated polymers.

Acknowledgment. The authors thank Michael Jones for experimental assistance.

Received: June 30, 2009 Accepted: August 15, 2009 Published: October 2, 2009

\section{REFERENCES}

1. G. E. Ashby, J. Polym. Sci., 50, 99 (1961).

2. J. L. Bolland and G. Gee, Trans. Faraday Soc., 42, 236 (1946).

3. P. K. Fearon, S. W. Bigger, and N. C. Billingham, J. Therm. Anal. Calorim., 76, 75 (2004).

4. I. Blakey, B. Goss, and G. George, Aust. J. Chem., 59, 485 (2006).

5. L. Matisova-Rychla and J. Rychly in "Recent Developments in Polymer Research,” A. V. Hopper Ed., Nova Science, New York,
2007, p. 163.

6. K. Jacobson, P. Eriksson, T. Reitberger, and B. Stenberg, Adv. Polym. Sci., 169, 151 (2004).

7. J. M. Gromek and M. R. Derrick, Polym. Degrad. Stab., 39, 261 (1993).

8. Z. Osawa, F. Konoma, S. Wu, and J. Cen, Polym. Photochem., 7, 337 (1986).

9. A. L. Margolin and V. Y. Shlyapintokh, Polym. Degrad. Stab., 66, 279 (1999).

10. J. Cen, F. Konoma, and Z. Osawa, Polym. Photochem., 7, 469 (1986).

11. H. Kihara, T. Yabe, and S. Hosada, Polym. Bull., 29, 369 (1992).

12. G. A. George, G. T. Egglestone, and S. Z. Riddell, Polym. Eng. Sci., 23, 412 (1983).

13. K. R. Millington, C. Deledicque, M. J. Jones, and G. Maurdev, Polym. Degrad. Stab., 93, 640 (2008).

14. F. Gugumus, in "Plastics Additives Handbook," 4th ed., R. Gacher and H. Muller Ed., Hanser, Cincinnati, 1993, chap. 3, p. 129.

15. T. Karstens and V. Rossbach, Makromol. Chem., 190, 3033 (1989).

16. T. Karstens and V. Rossbach, Makromol. Chem., 191, 757 (1990).

17. K. R. Millington, Color. Technol., 122, 169 (2006).

18. A. Plonka, Prog. React. Kinet. Mech., 16, 157 (1991).

19. D. J. Carlsson, C. J. B. Dobbin, and D. M. Wiles, Macromolecules, 18, 1791 (1985).

20. R. Basheer and M. Dole, J. Polym. Sci., Part B: Polym. Phys., 21, 111 (1983).

21. R. Basheer and M. Dole, Int. J. Chem. Kinet., 13, 1143 (1981).

22. M. Dole, Radiat. Phys. Chem., 31, 595 (1988).

23. M. Dole, J. Phys. Chem., 91, 3117 (1987).

24. M. Nurul-Huda, H. Dragaun, S. Bauer, H. Muschik, and P. Skalicky, Colloid Polym. Sci., 263, 730 (1985).

25. I. Gruszka, S. Lewandowski, E. Benko, and M. Perzyna, Fibres Text. East Eur., 13, 133 (2005).

26. N. Reddy and Y. Yang, J. Polym. Environ., 15, 81 (2007).

27. S.-S. Gouk, N. M. Kocherginsky, Y. Y. Kostetski, H. O. Moser, P. Yang, T.-M. Lim, and W. Q. Sun, Radiat. Res., 163, 535 (2005).

28. Y. Hori, S. Shimada, and H. Kashiwabara, J. Polym. Sci., Part B: Polym. Phys., 22, 1407 (1984). 